\title{
Prevalence of blindness and cataract surgery in Nepal
}

\author{
G P Pokharel, G Regmi, S K Shrestha, A D Negrel, L B Ellwein
}

\begin{abstract}
Background-A national eye care programme was launched in Nepal in the early 1980s. The impact of this programme on blindness and cataract surgery prevalence was evaluated in two geographic zones.

Methods-People aged 45 years and older were sampled using a stratified cluster design. Within randomly selected clusters, door to door enumeration was followed by visual acuity measurement and eye examinations at conveniently located sites. The full survey was preceded by a pilot study where operational methods were refined and quality assurance measures carried out.

Results-Of the 5112 enumerated individuals $90 \%$ were examined. Blindness, defined as presenting visual acuity less than $6 / 60$ in both eyes, was found in $5.3 \%$ $(95 \%$ CI $3.6,6.8)$ of individuals examined, with cataract being the principal cause in at least one eye in $78 \%$ of cases. Considering both cataract operated and unoperated cataract blind cases, surgical coverage was approximately $42 \%$.

Conclusion-The findings suggest that blindness prevalence may have decreased slightly from that estimated in a 1981 national survey, both overall and cataract related. Similarly, cataract surgical coverage may have increased somewhat. None of these changes, however, are at statistically significant levels. Accordingly, the blindness problem remains challengingly high.
\end{abstract} (Br F Ophthalmol 1998;82:600-605)

Organisation, Geneva, Switzerland

A D Negrel

National Eye Institute, Bethesda, Maryland, USA

L B Ellwein

Correspondence to: Leon B Ellwein, PhD, National Eye Institute, 31 Center Drive, MSC 2510, Bldg 31, Rm 6A-04, Bethesda, MD 20892-2510, USA.

Accepted for publication 9 January 1998 gramme was launched with a two pronged strategy: (1) development of training programmes for eye care professionals to staff hos- pitals and eye care centres, (2) establishment of primary, secondary, and tertiary eye care centres in areas where services were most in need. For both of these activities, the national eye care programme obtained substantial support from international non-governmental organisations. Nepal Netra Jyoti Sangh (NNJS), a local non-governmental organisation, was established to take responsibility for developing eye care services in Nepal, including the coordination of services provided by the different organisations operating in the various parts of the country. By the end of 1994, an estimated 140000 surgeries (primarily cataract) had been performed in Nepal; however, a significant portion were in patients from neighbouring India.

The current evaluation was motivated by an interest in assessing the impact of the NNJS coordinated blindness prevention efforts over the past 15 years. The evaluation was carried out in 1995 in two of the 14 zones of the countrynamely, Lumbini in the western region and Bheri in the mid-western region. These two zones were singled out for evaluation because of the record of accomplishment of two local hospitals in addressing the objectives of the national programme. Eye care services in the Lumbini zone started in 1985 with the active participation and support of the SEVA foundation in establishing the Rana-Ambika Eye Hospital, while services in Bheri zone started in 1983 with the efforts and support of the Swiss Red Cross Society in establishing the Fateh-Bal Hospital in Nepalgunj. The objectives of the evaluation were threefold: (1) estimate the current prevalence of blindness (and that due to cataract) in Lumbini and Bheri zones; (2) assess the impact of Lumbini and Bheri eye care programmes by comparing cataract blindness prevalence with that from the 1981 Nepal survey; (3) assess the impact of cataract surgery on visual functioning and quality of life as perceived by the patient. This paper addresses study methods and results dealing with blindness prevalence, the first two objectives. Cataract surgery outcomes are addressed in a companion paper. $^{2}$

\section{Methods}

STUDY DESIGN AND SAMPLING FRAME

The survey target population was all people aged 45 years or over in the Lumbini and Bheri 
zones of Nepal. (The 45 year lower age limit coincided with the introduction of democracy in Nepal in 1950; this well known event was used to help determine who met the age requirement among those not aware of their exact date of birth.) The study sample was divided evenly between the two zones. Each sample was in turn allocated proportionately to districts within the respective zone based on 1995 population estimates derived from 1991 census data. This resulted in the Lumbini zone being divided into six geographic strata (corresponding to its six districts) and the Bheri zone into five strata.

Each district is partitioned into rural village development councils (VDCs), and urban municipalities, which are then further subdivided into nine wards for VDCs and nine or more wards for municipalities. The district sample was allocated to urban and rural strata based on the proportionate populations. A listing of all wards within each district was prepared by first listing the VDCs and municipalities in alphabetical order, keeping the urban and rural strata separate, and then enumerating the individual wards within each VDC and municipality in numerical order.

Within each district, cluster sampling was employed, where clusters were defined using wards. Ward clusters were selected randomly with probability proportionate to size from the urban and rural strata separately within each district. The desired cluster size was approximately 100 people $\geqslant 45$ years of age (a 1 day eye examination workload). However, because there was considerable variation in ward sizes, small selected wards were combined with adjacent ones and large ones were subdivided. This combining and subdividing was carried out as follows. If the size of a selected ward was estimated as having less than 75 people age 45 years or older, it was combined with the next ward on the ordered listing (with the cluster now consisting of both wards). If the next listed ward was from a different VDC, the first listed ward within the same VDC was chosen for the combining. If a selected ward was estimated to be larger than 150 people, it was subdivided into two or more sampling units of approximately equal size and one selected randomly.

\section{SAMPLE SIZE CALCULATION}

The sample size calculation was based on estimating cataract blindness $(<3 / 60$ in both eyes) in those aged 45 years or older within an error bound of $25 \%$ with $95 \%$ confidence. The a priori estimate of cataract blindness prevalence, based on the 1981 Nepal survey, was taken to be $3.0 \%$.

With simple random sampling, the required sample size was calculated as 1989. This number was adjusted upward by a factor of 2.0 to account for cluster sampling design effects and adjusted further to account for a possible $15 \%$ non-response rate. Accordingly, the final sample size estimate was 4680 . With an average cluster size of 100 people $\geqslant 45$ years of age, 48 clusters were randomly selected, 24 from each zone.
The clusters actually selected ranged in estimated size from 86 to 150 people $\geqslant 45$ years of age in the Lumbini zone and from 75 to 148 people in the Bheri zone. In the Lumbini zone it was necessary to subdivide nine of the selected wards and combine five. In Bheri, 10 were subdivided and five combined. Of the 24 clusters selected from each zone, four in Lumbini and two in Bheri were urban.

FIELD PROCEDURES

Field work consisted of visiting all households within the cluster (village) to enumerate all people aged 45 years or over, followed by visual acuity measurements, visual functioning (VF) and quality of life (QOL) interviews, and eye examinations at a nearby location. The work was carried out by two different field teams, one in each zone. Each team consisted of an ophthalmologist, two ophthalmic assistants, two interviewers, and six enumerators (two were supervisors), plus a driver and logistical aid. The enumerators were divided into two subteams, each with two enumerators and a supervisor. An enumeration subteam visited the cluster in advance of the clinical personnel and stayed until the clinical work was completed. Thus, the need for two enumeration subteams.

All study subjects (people aged $\geqslant 45$ years) were given a referral slip and asked to come to the village examination site on a particular date, usually a day or two after the enumeration. Literacy was determined by asking the subject whether he/she can read and write. Study subjects appearing at the examination site first underwent visual acuity measurement by one of the two ophthalmic assistants. Presenting visual acuity was assessed in each eye using a WHO tumbling $\mathrm{E}$ test card. Pinhole vision was measured in those eyes with presenting visual acuity $<6 / 18$. (The vision acuity measurement procedure was the same as that used in the 1981 survey.)

All aphakics and pseudophakics, those with presenting visual acuity $<6 / 60$ in either eye, and a one in 20 sample of those with presenting vision $\geqslant 6 / 18$ in both eyes (normal or near normal) were interviewed by one of the two VF/QOL interviewers.

All participants received a basic eye examination of the eyelid, globe, pupillary reflex, and lens status by the team ophthalmologist using a torch light, $2 \times$ binocular loupe, and hand held slit lamp. Optic disc and retinal blood vessels were examined by direct ophthalmoscope. Additionally, detailed examination of media and fundus was performed with dilated pupil on those whose vision did not improve to $\geqslant 6 / 18$ on pinhole with slit lamp and direct ophthalmoscope. Intraocular pressure was measured by an ophthalmic assistant using a Perkin hand held applanation tonometer.

Refraction was carried out by slit retinoscope by an ophthalmic assistant whenever presenting vision was less than $6 / 18$ and in all aphakics and pseudophakics, as well as those requesting presbyopic correction. Full refraction including retinoscopy and subjective verification with spherical and astigmatic 
Table 1 Enumerated and examined study population by age, sex, and literacy (\%)

\begin{tabular}{|c|c|c|c|}
\hline & \multicolumn{3}{|c|}{ Study population } \\
\hline & Enumerated & No examined & $\%$ Examined \\
\hline \multicolumn{4}{|l|}{ Age: } \\
\hline $45-49$ & 1519 (29.7) & $1386(30.1)$ & 91.2 \\
\hline $50-59$ & $1769(34.6)$ & $1561(33.9)$ & 88.2 \\
\hline $60-69$ & $1146(22.4)$ & $1033(22.4)$ & 90.1 \\
\hline $70+$ & $678(13.3)$ & $622(13.5)$ & 91.7 \\
\hline \multicolumn{4}{|l|}{ Sex: } \\
\hline Male & $2595(50.8)$ & $2253(49.0)$ & 86.8 \\
\hline Female & $2517(49.2)$ & $2349(51.0)$ & 93.3 \\
\hline \multicolumn{4}{|l|}{ Literacy: } \\
\hline Literate & $1102(21.6)$ & 915 (19.9) & 83.0 \\
\hline Illiterate & $4010(78.4)$ & $3687(80.1)$ & 91.9 \\
\hline All & $5112(100.0)$ & $4602(100.0)$ & 90.0 \\
\hline
\end{tabular}

correction was used in establishing best corrected visual acuity.

Oral informed consent was obtained during the house to house enumeration. Those with visual acuity $<6 / 60$ in both eyes because of cataract were offered free surgery. Standard +10 dioptre aphakic glasses were provided to all aphakics free of cost. High myopes were given prescriptions and asked to collect glasses free of cost from the zone eye hospital. Medical treatment for minor eye abnormalities (for example, conjunctivitis, dry eye) was provided in the field. Those needing further treatment were referred to the zone eye hospital.

STAFF TRAINING

Field enumerators were oriented to the study protocol and objectives, and trained in techniques for household mapping over a 3 week period in Kathmandu. A detailed field manual was prepared and provided to each enumerator.

Two experienced VF/QOL interviewers from Aravind Eye Hospital in Madurai, India conducted 2 weeks of interviewer training, initially in Kathmandu, followed by 4 days of team training at Fateh-Bal Eye Hospital in Bheri zone. Interviewers were oriented on the structure and content of the VF/QOL questionnaire. Interviewer training included role playing followed by practice interviews on outpatients and inpatients at the Nepal Eye Hospital in Kathmandu. Interviewing methods and difficulties were reviewed in problem solving sessions.

One week of training was carried out for the ophthalmic assistants at Fateh-Bal Hospital in Bheri zone. This training included instruction on the principles of an eye examination, including practice with visual acuity and intraocular pressure measurement. They were also oriented to dealing with populations in field settings.

One week of training, also at Fateh-Bal Eye Hospital, was conducted for the two team ophthalmologists. This included review of the study protocol and objectives, with emphasis on the content and rationale behind the clinical examination data form. Practice in completing the examination form was carried out using hospital patients. Use of a hand held slit lamp was also practised.

\section{PILOT STUDY}

Upon completion of training, a pilot study was carried out in three rural wards in Bheri zone. These wards were not among those selected for the main survey. Staff from both field teams participated together in the pilot. In all, 289 people aged $\geqslant 45$ years from 299 households were enumerated and 259 people were examined. The pilot study provided a field test of all study procedures, completion of data collection forms and patient logistics. The pilot study was also used to ensure that the training of ophthalmic assistants, interviewers and ophthalmologists was adequate, based on assessments of interobserver variation for critical data items.

The two ophthalmic assistants from each of the two teams were compared with each other during the pilot in the measurement of presenting visual acuity in 50 patients, half with visual acuity $<6 / 18$ in at least one eye; agreement was satisfactory for both teams. Interobserver variation was monitored again at mid-study across the visual acuity range to ensure that any drift between the two assistants would not go unrecognised. The kappa statistics were 0.75 and 1.00 (left eyes and right eyes respectively) in Bheri zone ( 35 cases) and 0.72 and 0.66 in Lumbini (49 cases), indicating good agreement between ophthalmic assistants in measuring visual acuity.

Each of the two study ophthalmologists was compared with a senior ophthalmologist (GPP) with respect to agreement in diagnosing refractive error and cataract as the principal cause of low vision (or blindness) in 47 cases. Substantial discrepancies were noted, which led to a clarification of diagnostic criteria. During the survey,

Table 2 Visual impairment and blindness prevalence by age, sex, and literacy based on presenting visual acuity (\%)

\begin{tabular}{|c|c|c|c|c|c|c|}
\hline & $\begin{array}{l}\text { Normal/ near } \\
\text { normal }\end{array}$ & $\begin{array}{l}\text { Visual } \\
\text { impairment }\end{array}$ & $\begin{array}{l}\text { Unilateral } \\
\text { blindness }\end{array}$ & $\begin{array}{l}\text { Moderate } \\
\text { blindness }\end{array}$ & $\begin{array}{l}\text { Severe } \\
\text { blindness }\end{array}$ & $\begin{array}{l}\text { Missing visual } \\
\text { acuity }\end{array}$ \\
\hline \multicolumn{7}{|l|}{ Age: } \\
\hline $45-49$ & $1244(89.8)$ & $62(4.5)$ & $50(3.6)$ & $7(0.5)$ & $11(0.8)$ & $12(0.9)$ \\
\hline $50-59$ & $1264(81.0)$ & $164(10.5)$ & $85(5.4)$ & $21(1.3)$ & $21(1.3)$ & $6(0.4)$ \\
\hline $60-69$ & $643(62.2)$ & $211(20.4)$ & $95(9.2)$ & $40(3.9)$ & $38(3.7)$ & $6(0.6)$ \\
\hline $70+$ & $228(36.7)$ & $179(28.2)$ & $110(17.7)$ & $37(5.9)$ & $66(10.6)$ & $2(0.3)$ \\
\hline \multicolumn{7}{|l|}{ Sex: } \\
\hline Male & $1662(73.8)$ & $314(13.9)$ & $165(7.3)$ & $46(2.0)$ & $52(2.3)$ & $14(0.6)$ \\
\hline Female & $1717(73.1)$ & $302(12.9)$ & $175(7.4)$ & $59(2.5)$ & $84(3.6)$ & $12(0.5)$ \\
\hline \multicolumn{7}{|l|}{ Literacy: } \\
\hline Literate & $767(83.8)$ & $85(9.3)$ & $39(4.3)$ & $5(0.5)$ & $13(1.4)$ & $6(0.7)$ \\
\hline Illiterate & $2612(70.8)$ & $531(14.4)$ & $301(8.2)$ & $100(2.7)$ & $123(3.3)$ & $20(0.5)$ \\
\hline All & $3379(73.4)$ & $616(13.4)$ & $\begin{array}{l}340(7.4) \\
(5.22-9.55)^{\star}\end{array}$ & $\begin{array}{l}105(2.3) \\
(1.52-3.04)^{\star}\end{array}$ & $\begin{array}{l}136(3.0) \\
(2.05-3.86)^{\star}\end{array}$ & $26(0.6)$ \\
\hline All (best corrected) & $3839(83.4)$ & $279(6.1)$ & $\begin{array}{l}280(6.1) \\
(4.30-7.87)^{\star}\end{array}$ & $\begin{array}{l}65(1.4) \\
(0.94-1.88)^{\star}\end{array}$ & $\begin{array}{l}113(2.5) \\
(1.70-3.21)^{\star}\end{array}$ & $26(0.6)$ \\
\hline
\end{tabular}

$\star 95 \%$ confidence intervals. 
Table 3 Principal cause of blindness for individuals with moderate or severe bilateral blindness

\begin{tabular}{|c|c|c|c|c|c|c|c|c|}
\hline \multirow[b]{2}{*}{ Right eye } & \multicolumn{8}{|l|}{ Left eye } \\
\hline & $\begin{array}{l}\text { Refractive } \\
\text { error }\end{array}$ & $\begin{array}{l}\text { Uncorrected } \\
\text { aphakia }\end{array}$ & Cataract & Trachoma & $\begin{array}{l}\text { Corneal } \\
\text { scar }\end{array}$ & Glaucoma & $\begin{array}{l}\text { Other and } \\
\text { missing }\end{array}$ & Total \\
\hline Refractive error & 4 & & 2 & & 1 & & & 7 \\
\hline Uncorrected aphakia & & 3 & 3 & & 1 & & 3 & 10 \\
\hline Cataract & 3 & 4 & 144 & & 1 & & 10 & 162 \\
\hline Trachoma & & & & 7 & & & 2 & 9 \\
\hline Corneal scar & & & 3 & & 2 & & 3 & 8 \\
\hline Glaucoma & & & & & & 4 & 1 & 5 \\
\hline Other and missing & 1 & 1 & 18 & 2 & & & 18 & 40 \\
\hline Total & 8 & 8 & 170 & 9 & 5 & 4 & 37 & 241 \\
\hline
\end{tabular}

the Bheri zone ophthalmologist resigned and a replacement was employed. This replacement underwent interobserver comparison with the senior ophthalmologist, including 17 refractive error and nine cataract eyes, with complete agreement. Midterm evaluation in the Lumbini zone, including seven refractive error and 25 cataract eyes, also yielded complete agreement between ophthalmologists.

DATA MANAGEMENT

Completed data collection forms were edited for missing values and accuracy in the field. Problems were rectified to the extent possible by the responsible team member. Edited forms were packaged and sent to Kathmandu where final editing and computerised data entry using FOX PRO software took place. All data were entered twice by two independent data clerks and discrepancies detected by a matching program were resolved. Final data cleaning included value frequency analysis and range checks. The cleaned data sets were translated into system files for statistical analysis using sPSs/4.0 software.

\section{STATISTICAL ANALYSIS}

Five vision categories were defined using visual acuity cut offs from the 1981 survey: (1) normal or near normal vision, $\geqslant 6 / 18$ in both eyes; (2) visual impairment, $<6 / 18$ to $\geqslant 6 / 60$ in worse eye, $\geqslant 6 / 60$ in better eye; (3) unilateral blindness, $<6 / 60$ in worse eye, $\geqslant 6 / 60$ in better eye; (4) moderate bilateral blindness, $<6 / 60$ in worse eye, $<6 / 60$ to $\geqslant 3 / 60$ in better eye; (5) severe bilateral blindness, $<3 / 60$ in both eyes. Because these categories do not correspond precisely with those defined in the World Health Organisation's international classification of diseases, different category terminology was used to avoid confusion. Estimates (with 95\% confidence

Table 4 Prevalence of aphakia/pseudophakia by age, sex, and literacy

\begin{tabular}{ll}
\hline & Total No of operated cases (\%) \\
\hline Age: & \\
$45-49$ & $11(6.9)$ \\
$50-59$ & $27(17.0)$ \\
$60-69$ & $45(28.3)$ \\
$70+$ & $76(47.8)$ \\
Sex: & $65(40.9)$ \\
$\quad$ Male & $94(59.1)$ \\
Female & \\
Literacy: & $21(13.2)$ \\
$\quad$ Literate & $138(86.8)$ \\
Illiterate & $159(100.0)$ \\
All & \\
\hline
\end{tabular}

intervals) of impairment and blindness prevalence were calculated along with that attributed specifically to cataract.

The prevalence of aphakia and pseudophakia is also of interest, particularly as it pertains to surgical coverage, which is defined as the number of bilaterally blind cataract cases operated divided by the number who could have been operated. The denominator includes the already operated bilaterally blind (the numerator) plus the unoperated bilaterally blind with cataract the principal cause of blindness in at least one eye. The surgical coverage calculation is imprecise because without the preoperative visual acuity of both eyes it is not possible to know for sure whether a cataract operated case was blind bilaterally at the time of surgery. At the risk of overstating surgical coverage, an operated case is included in the numerator unless it is impossible for it to have been blind before surgery. Accordingly, cases are excluded if one eye is unoperated and has visual acuity that exceeds the blindness category definition. Cases operated in both eyes are always included.

Logistic regression was used to address the likelihood of receiving cataract surgery as a function of various sociodemographic variables.

\section{Results}

A total of 5112 people $\geqslant 45$ years of age were enumerated in the 48 randomly selected ward clusters (Table 1). Examinations were conducted on 4602 cases- 340 of those enumerated were not available for examination because of temporary absence from the area and another 170 refused to cooperate. This $90.0 \%$ response rate was better than expected; the response was $88.4 \%$ in the Lumbini zone and $91.9 \%$ in Bheri zone. The lower response in Lumbini zone is explained by a greater number being unavailable, rather than refusal to cooperate.

The difference between the examined population and the census population is not statistically significant with regard to age percentages (goodness of fit $\chi^{2}=0.88, \mathrm{p}=0.83$ ) or sex percentages $\left(\chi^{2}=0.32, \mathrm{p}=0.57\right)$. Census data on literacy are not available for comparison.

Table 2 presents prevalence estimates of visual impairment and blindness based on presenting visual acuity. A resampling jack knife method was used to calculate confidence intervals around point estimates of prevalence. ${ }^{3}$ The prevalence and $95 \%$ confidence interval for 
Table 5 Surgical coverage by age, sex, and literacy under two alternative assumptions regarding the degree of blindness at the time of being operated

\begin{tabular}{|c|c|c|}
\hline & \multicolumn{2}{|c|}{ Preoperative blindness } \\
\hline & Severe & Severe and moderate \\
\hline \multicolumn{3}{|l|}{ Age: } \\
\hline $45-49$ & $71.4(10 / 14)^{\star}$ & $50.0(10 / 20)$ \\
\hline $50-59$ & $60.7(17 / 28)$ & $45.0(18 / 40)$ \\
\hline $60-69$ & $60.7(34 / 56)$ & $38.5(37 / 96)$ \\
\hline $70+$ & $53.9(55 / 102)$ & $42.5(59 / 139)$ \\
\hline \multicolumn{3}{|l|}{ Sex: } \\
\hline Male & $63.3(50 / 79)$ & $44.2(53 / 120)$ \\
\hline Female & $54.6(66 / 121)$ & $40.6(71 / 175)$ \\
\hline \multicolumn{3}{|l|}{ Literacy: } \\
\hline Literate & $63.6(14 / 22)$ & $57.1(16 / 28)$ \\
\hline Illiterate & $57.3(102 / 178)$ & $40.5(108 / 267)$ \\
\hline All & $58.0(116 / 200)$ & $42.0(124 / 295)$ \\
\hline
\end{tabular}

*Within the parenthesis, the numerator represents the estimated number of cataract operated cases meeting the preoperative blindness assumption and the denominator represents the sum of this number plus the number of unoperated, still blind, cases.

moderate and severe bilateral blindness together is $5.3 \%(3.6,6.8)$. Using best corrected instead of presenting visual acuity to define blindness, the combined prevalence and 95\% confidence interval is $3.9 \%(2.7,5.0)$.

Visual impairment and blindness prevalence are clearly related to increasing age. Thus, in exploring whether there are significant differences in blindness across sex or literacy subgroups, adjustment for age differences between the subgroups is necessary. After adjusting for age differences between males and females in the examined population (females are somewhat younger, which is consistent with census data), the observed difference in prevalence increases; this higher prevalence in females is statistically significant $\left(\chi^{2}=14.5, p=0.006\right)$. The higher prevalence of impairment and blindness among the illiterate is also statistically significant $(\mathrm{p}<0.001)$.

The principal cause of blindness by eye for those individuals categorised as having either moderate or severe blindness is shown in Table 3. Age related cataract is the dominant cause of blindness in this age group: 188 (78\%) individuals are blind, with cataract being the principal cause in at least one eye.

Unilaterally blind individuals also suffer from cataract as the most common cause. Out of the 340 individuals, $158(46.5 \%)$ have cataract as the principal cause of impairment in the blind eye (data not shown). Another 13 eyes $(3.8 \%)$ were blind with refractive error as the principal cause. This results in 171 unilaterally blind individuals $(50.3 \%)$ who can potentially be helped by a combination of glasses and cataract surgery.

For those categorised as having vision impairment $(<6 / 18$ to $\geqslant 6 / 60$ in one or both eyes), $400(64.9 \%)$ had refractive error identified as the principal cause in at least one eye and $184(29.9 \%)$ with cataract as the principal cause in at least one eye (data not shown). Twenty eight $(4.5 \%)$ of these individuals had both of these two causes identified-that is, refractive error in one eye and cataract in the other eye. Although not blind in either eye, $90.3 \%$ could potentially benefit from glasses or cataract surgery.
Table 4 presents the cataract operated cases by age, sex, and literacy. Of the 159 cases, 57 $(36.8 \%)$ were operated in both eyes. Twenty six of the cases $(16.3 \%)$ were pseudophakics. (None of the cases were pseudophakic in one eye and aphakic in the other.) Forty three per cent of cases were operated in surgical eye camps-based on the most recent surgery for those bilaterally operated.

Age, sex, literacy, zone and urban/rural area were investigated as predictors of cataract surgery among the 4602 cases examined. Beyond the obvious influence of advancing age, female sex (Wald test, odds ratio $=1.6, \mathrm{p}=0.0112$ ), Bheri zone (odds ratio $=2.0, \mathrm{p}<0.0001$ ), and urban area (odds ratio $=2.3, \mathrm{p}=0.0002$ ) are predictors of cataract surgery at statistically significant levels.

The above analysis does not take into account differences across the sociodemographic subgroups in the underlying likelihood of having cataract. This is explored for age, sex, and literacy by considering surgical coverage, where the number of cases operated are adjusted by the number of individuals who were actual candidates for cataract surgery. The surgical coverage data presented in Table 5 are based on two alternative assumptions: the most relaxed assumption is that cases were not operated until they were severely blind, unless there is contrary evidence, such as unilaterally operated cases with visual acuity $>3 / 60$ in the fellow eye. Under this assumption, surgical coverage among the severely blind is $58.0 \%$. The more conservative assumption is that operated cases may have been only moderately blind at the time of cataract surgery. With this (perhaps more realistic) assumption, surgical coverage drops to $42.0 \%$. In both scenarios, the surgical coverage presented should be considered an upper estimate, because we have no way of knowing whether all of the operated cases that now meet the particular inclusion criteria did so at the time of surgery. For example, a bilaterally operated case may have been no more than unilaterally blind at the time of first being operated and, thus, should be excluded from the surgical coverage calculations.

The analyses suggest that surgical coverage increases as a function of younger age, male sex, and literacy. (Because the numbers are depended heavily on the assumptions, they have not been subjected to statistical testing.) The previous analysis showing that females have higher odds of being operated for cataract surgery than males is not explained by a correspondingly higher surgical coverage. In fact, because of the more than offsetting higher prevalence in females, the opposite holds. The same applies for advancing age, where the elderly clearly have a higher prevalence.

The surgical coverage in Bheri zone was $67.3 \%$ and $50.3 \%$, under the severe blindness and moderate blindness assumptions, respectively; the coverages in Lumbini were $46.0 \%$ and $31.8 \%$, respectively (data not shown). For both zones, the coverage in urban areas was higher than that in rural areas. 


\section{Discussion}

Within zones, the cluster sampling was consistent with a probability proportional to size approach until the final adjustment, where small selected ward clusters were combined with adjacent ones and large wards were subdivided. This resulted in a bias where an individual living in a ward adjacent to a small one had an increased chance of being included in the survey and one living in a large ward a reduced chance. Further, because the sample was equally distributed between the two zones, individuals in Bheri (the smaller of the two) had a slightly increased chance over those in Lumbini of being in the survey. Although no attempt has been made to make adjustments in the findings to account for any of these imbalances, there is no apparent reason to believe that the results are not representative in spite of the cluster size adjustments.

The $95 \%$ confidence intervals are larger than the desired error bound used in the sample size calculation. Although the sample size was doubled to account for the cluster design effect, apparently this was insufficient. The between cluster variation was considerable, in that some clusters had a severe blindness prevalence over $10 \%$, while it was zero in others.

The higher than expected examination response rate among enumerated people was realised across all sociodemographic subgroups. Non-responders were more likely to be male and literate than responders, although these differences were small. Information is not available to judge whether the visual status of non-responders differs from that of responders. Although the thoroughness of the enumeration was not independently verified, the two enumerators on each subteam worked as a pair, not independently, allowing for work cross checking. Because the enumerators were well qualified, having had several years prior experience in similar studies, there is every reason to believe that they were exceedingly thorough.

A higher prevalence of blindness in women is consistent with that found in $1981 .{ }^{1}$ Reduced access to cataract surgery is a contributing factor, as suggested by surgical coverage data. But it is not sufficient. A higher risk of cataract appears to be another reason. Combining cataract operated with unoperated severe and moderate cataract blindness cases (the denominator of the parenthetical fraction shown in Table 5) and dividing by the number of cases examined produces a higher cataract burden among females (74/1000 females versus 53/ 1000 males) in spite of their being younger than males. The same holds for the illiterate population, where the difference is even more pronounced $(72 / 1000$ versus $31 / 1000)$. This is only partly explained by an illiterate population that is $61 \%$ female and older than the literates.

In the surgical coverage analysis it was necessary to exclude several unilateral operated cases where the unoperated eye has good vision, because they could not have been bilaterally blind at the time of surgery. Some of these cases are aphakic (as opposed to pseudophakic with IOL implantation) and are probably experiencing image fusion problems if vision was restored in the operated eye. With the increasing availability of low cost IOLs, we would expect that intracapsular cataract extraction with aphakic spectacles will no longer be practised in the unilaterally blind, because of the less than satisfactory vision result. ${ }^{4}$

As evidenced by Table 3, most of the blind can be potentially helped by either glasses for refractive error and uncorrected aphakia or by cataract surgery (202 individuals out of the 241 blind). The extent to which cataract surgery has been successful in restoring vision is presented in a companion paper, which deals with not only the clinical visual acuity outcome but also visual functioning and related quality of life as reported by the patient. ${ }^{2}$

The survey findings suggest that blindness prevalence may have decreased since 1981, both overall and cataract related. Severe blindness (all causes) was estimated as $3.0 \%$ versus approximately $3.8 \%$ in the 1981 Nepal-wide survey. Any rigorous comparison of 1995 blindness prevalence in Lumbini and Bheri zones with 1981 rates would require calculation of age sex standardised rates to account for an aging population and possible changes in sex distribution between 1981 and 1995. However, even without such adjustments to crude rates, which would only narrow the difference, it is evident from the $2.05 \%$ to $3.86 \%$ confidence interval around the $19953.0 \%$ point estimate that the reduction, if any, is insufficient to be of statistical significance. (Accordingly, no further analyses were attempted.) If a small reduction in blindness has, indeed, been realised, an increase in cataract surgery may be chiefly responsible. Cataract surgery coverage within Bheri and Lumbini zone in 1981 was approximately $44 \%$ among those with severe blindness (Brilliant et al ${ }^{1}, \mathrm{p}$ 215), while the corresponding percentage in 1995 was $58.0 \%$.

Whatever the impact of prevention of blindness over the past 15 years might have been, it is abundantly clear that the blindness problem, and that due to cataract specifically, remains at challengingly high levels.

Work supported in part by the World Health Organisation under National Institutes of Health contract N01-EY-2103.

The authors wish to thank Marriam Bright, National Eye The authors wish to thank Marriam Bright, National Eye
Institute, for clerical assistance in preparing this manuscript. Institute, for clerical assistance in preparing this manuscript.
Thanks are also due to Dr R P Pokharel, Nepal Netra Jyoti Sangh, and to staff at the Fatah-Bal and Rana-Ambika hospitals Sangh, and to staff at the
for facilitating the study.

1 Brilliant GE, Pokharel RP, Grasset NC, et al. The epidemiology of blindness in Nepal: report of the 1981 nepal blindness survey. Chelsea, MI: The Seva Foundation, 1988.

2 Pokharel GP, Selvaraj S, Ellwein LB. Visual functioning and quality of life outcomes among cataract operated and unoperated blind populations in Nepal. Br $\mathcal{f}$ Ophthalmol unoperated blind

3 Cochrane W. Sampling techniques. 3rd ed. New York: J Wiley, 1984:178.

4 Ellwein LB, Kupfer C. Strategic issues in preventing blindness in developing countries. Bull World Health Organ 1995; 73:681-90. 